\title{
WERNER TROSSBACH
}

\section{TAGELÖHNER UND DÖRFLICHE ELITEN}

\author{
Überlegungen zum Verhältnis von Struktur- und Ereignisgeschichte \\ im Bauernkrieg (1524-1526)
}

Bereits die Zeitgenossen waren sich nicht schlüssig, ob sie die Erhebung der Jahre 1524-1526 einen Bauernkrieg oder einen Aufstand »des gemeinen Mannes in Stadt und Land " ${ }^{1}$ nennen sollten. Der gesamtgesellschaftliche Charakter spiegelt sich z.B. im Aufstandsgeschehen wider, von dem in weiten Gebieten Stadt und Land erfaßt wurden, und in dem Personenkreis, der in den sogenannten Bauernhaufen Führungsaufgaben wahmahm. Neben Städtebewohnern fand sich darunter auch eine Anzahl von Lokalbeamten und Angehörigen des niederen Adels. Demgegenüber darf allerdings nicht vergessen werden, daß anspruchsvolle Organisationsaufgaben auch von zahlreichen Menschen vollbracht wurden, die zu den bäuerlichen Gemeinden gehörten ${ }^{2}$. Die Widersprüche, die sich aus der uneinheitlichen Zusammensetzung der Bewegung und besonders der Führungsgruppen ergaben, konnten wegen der raschen und nachhaltigen Niederlage kaum ausgetragen werden, daß sie aber wenigstens rudimentär angesprochen wurden, zeigen die Ressentiments, auf die Götz von Berlichingen, ein Condottiere aus dem niederen Adel, der sich nolens volens auf der Seite der Bauern wiederfand, im Lager vor Würzburg traf. Er wurde abgesetzt, weil er ain edelman wer. Seine Gegner stellten klar, sy hetten ein baurn krieg, sie wolten khein fürsten, graven, herrn oder edelman dabey haben ${ }^{3}$.

$\mathrm{Da}$ es - trotz der Teilnahme nichtbäuerlicher Schichten und einer im Verlauf immer stärker gesamtgesellschaftlich ausgerichteten Programmatik - tatsächlich zumindest im ersten Anlauf primär um die Interessenvertretung ländlicher Schichten ging, wird aus dem wichtigsten Dokument der Aufständischen, den Zwölf Artikeln, deutlich. In Übereinstimmung damit soll im folgenden die ländliche Seite der Erhebung von 1525, der Bauernkrieg im engeren Sinne des Wortes, im Mittelpunkt stehen, wobei sich ergeben wird, daß

\footnotetext{
${ }^{1}$ Peter BLICKLE, Die Revolution von 1525, München, Wien ${ }^{2} 1981$, S. 165-195.

${ }^{2}$ Günther FranZ, Die Führer im Bauemkrieg, in: DERS. (Hg.), Bauemschaft und Bauemstand 1500-1970, Limburg 1975 (Deutsche Fuhrungsschichten in der Neuzeit, 8), S. 1-15.

${ }^{3}$ Rudolf ENDRES, Probleme des Bauernkriegs in Franken, in: Rainer WoHLFEIL (Hg.), Der Bauernkrieg 1524-26, Munchen 1975, S. 108.
} 
auch diese nicht von einheitlichen Kräften geprägt wurde. Daß die zentrale Beschwerdeschrift, obwohl sie von Personen mit städtischem Erfahrungshintergrund, einem Kleriker und einem theologisch versierten Kürschner, abgefaßt worden war, allein Verhältnisse in der ländlichen Gesellschaft zur Sprache brachte und städtische Strukturen mit keinem Wort erwähnte, erklärt sich aus dem Zustandekommen der Artikel. Die Verfasser hatten Lokalbeschwerden verschiedener oberschwäbischer Bauernformationen ${ }^{4}$ lediglich systematisiert und mit theologischen Kommentaren versehen. Obwohl die Zwölf Artikel demgemäß nur in regionalem Maßstab ${ }^{5}$ Repräsentativität beanspruchen konnten, verbreiteten sie sich in Windeseile in den Bauernkriegsterritorien und darüber hinaus. Mittlerweile sind knapp dreißig Druckvarianten bezeugt ${ }^{6}$. Die Verfasser hatten offenbar mit ihren Kommentaren und Schlußfolgerungen einen Nerv getroffen, der die ländliche Bevölkerung auch andernorts elektrisierte, selbst wenn ein Teil der konkreten Beschwerden, wie es Herzog Georg von Sachsen für seinen Erfahrungsbereich zutreffend formulierte, der Thüringischen Landart ganz undienstlich und sich auf die Flecken und Dörfer gar nicht reimen und schicken werden?

Was die reale Lage der Aufständischen betrifft, so stimmt das Bild, das die Beschwerden vermitteln, mit hypothetischen Überlegungen überein, die an die Stelle der vielfach noch immer fehlenden Forschungen treten müssen. Neuere Darstellungen machen deutlich, daß die Pest im 14. Jahrhundert nicht überall mit der elementaren Wucht zugeschlagen hatte, die in den Arbeiten von Wilhelm Abel vorausgesetzt wird. Der Schwarze Tod hatte zwar auch Südwestdeutschland und Franken erreicht, dort aber zumindest in einigen Gebieten weniger verheerend gewütet als etwa im Nordosten des Reiches ${ }^{8}$. Von einer raschen demographischen Erholung zeugen z.B. Konflikte um Allmendenutzungen, die in württembergischen Dörfern bereits an der Wende zur Neuzeit festzustellen sind ${ }^{9}$. Wenngleich sich noch kein konsistentes Gesamtbild für die

\footnotetext{
${ }^{4}$ BLICKLE, Revolution (wie Anm. 1), S. 35-89.

${ }^{5}$ Selbst wenn man Einflüsse aus dem südlichen Oberrheingebiet (Peter BLICKLE, Nochmals zur Entstehung der Zwölf Artikel im Bauernkrieg, in: DERs. [Hg.], Bauer, Reich und Reformation. Festschrift für Günther Franz zum 80. Geburtstag, Stuttgart 1982, S. 286-308) einbezieht, ist damit nur ein kleiner Teil der Bauernkriegsgebiete als Ursprungsort der Zwölf Artikel zu benennen.

${ }^{6}$ Peter BLICKLE, Republiktheorie aus revolutionärer Erfahrung, in: DERS. (Hg.), Verborgene republikanische Traditionen in Oberschwaben, Tübingen 1998, S. 195-210, hier S. 210.

${ }^{7}$ Zit. nach: Günther FrANZ, Der deutsche Bauernkrieg, Darmstadt ${ }^{10} 1975$, S. 264. Eine Diskussion der »Anwendbarkeit« der Zwölf Artikel bei BLICKLE, Revolution (wie Anm. 1), S. 90-99.

${ }^{8}$ Manfred VASOLD, Die Ausbreitung des Schwarzen Todes in Deutschland nach 1348 . Zugleich ein Beitrag zur deutschen Bevölkerungsgeschichte, in: Historische Zeitschrift 277 (2003), S. 281-308.

${ }^{9}$ Paul WARDE, Law, the scommunes, and the Distribution of Resources in Early Modern German State Formation, in: Continuity and Change 17 (2002), S. 183-211.
} 
Kerngebiete des Bauernkrieges ergibt, müssen zumindest für die von den $\mathrm{Be}$ völkerungsverlusten weniger betroffenen Gebiete - etwa Ostschwabens ${ }^{10}-$ die Schlußfolgerungen überprüft werden, die für das 15 . Jahrhundert von einem Rückgang feudaler Renten und einem Verfall feudaler Macht ausgehen, wie er vor dem Hintergrund eines hohen Wüstungsquotienten tatsächlich für das Paderborner Land nachgewiesen worden ist ${ }^{11}$. Zumindest sollte in Betracht gezogen werden, daß ein solcher Verfall in den weniger betroffenen Gebieten des Südwestens spätestens zu Beginn des 16. Jahrhunderts weitgehend gestoppt war $^{12}$. In dieses Bild passen die Klagen, die in den Zwölf Artikeln und einer Vielzahl von lokalen Beschwerdeschriften in Franken, Oberschwaben und den Oberrheinlanden erhoben wurden. Sie beklagten Abgabenerhöhungen, Fron- und Steuerforderungen, auch Übergriffe auf die Allmende ${ }^{13} . \mathrm{Ob}$ man von einer réaction seigneuriale ${ }^{14}$ sprechen kann, müssen weitere Forschungen erweisen.

Auch im 15. Jahrhundert hatten die Herrschaftsträger des Südwestens einem wie auch immer ausgeprägten Verfall von Machtpositionen und Renten nicht tatenlos zugesehen. Sie trafen jedoch meist auf eine bäuerliche Gegenseite, deren Verhandlungsposition durch die Bevölkerungsverluste gestärkt war, wenn auch nicht in einem Maße, wie man es für manche Gebiete Nord- und Ostdeutschlands voraussetzen kann. Außerdem war es auch im Süden im 15. Jahrhundert für bäuerliche Betriebe noch möglich, gesteigerte Forderungen durch den Rückgriff auf freie Ressourcen zu kompensieren ${ }^{15}$. Nach 1500 hingegen waren im Südwesten die Grenzen des Wachstums zwar noch nicht er-

${ }^{10}$ Rolf KIESSLING, Der Schwarze Tod und die weißen Flecken, in: Konrad ACKERMANN, Hermann RUMSCHOTTEL (Hg.), Bayerische Geschichte/Landesgeschichte in Bayern, Festgabe für Alois Schmid, München 2005 (Zeitschrift für bayerische Landesgeschichte, 68), S. 519-539.

"Bruno H. LIENEN, Aspekte des Wandels bäuerlicher Betriebe zwischen dem 14. und dem 17. Jahrhundert an Beispielen aus Tudorf (Kreis Paderborn), in: Westfälische Forschungen 41 (1991), S. 288-315.

${ }^{12}$ Siehe die Überlegungen von David W. SABEAN, Landbesitz und Gesellschaft am Vorabend des Bauernkriegs, Stuttgart 1972, Michael B. W. GEYER, Sozial- und Besitzverhăltnisse unter geistlicher und weltlicher Grundherrschaft, dargestellt an den Besitzungen des Stifts Backnang und drei Strohgäudörfern unter württembergischer Herrschaft im Zeitraum von 1350-1545, Diss. Tübingen 1976 und Claudia ULBRICH, Leibherrschaft am Oberrhein im Spătmittelalter, Göttingen 1979 (Veröffentlichungen des Max-Planck-Instituts für Geschichte, 58). Skeptischer fur ein regionales Beispiel: Govind P. SREENIVASAN, The Peasants of Ottobeuren, 1487-1726, Cambridge 2004.

${ }^{13}$ Werner TrOSSBACH, Gemeinde und Allmende im deutschen Bauernkrieg. Annäherungen an die Denkweisen der Aufständischen, in: Zeitschrift für Agrargeschichte und Agrarsoziologie 49 (1992), S. 15-27.

${ }_{14}$ ULBRICH, Leibherrschaft (wie Anm. 12), S. 265, sieht bereits fulr das 15. Jahrhundert einen herrschaftlichen »Intensivierungsproze $ß$.

${ }^{15}$ Gowind P. SREEnIVASAN, The Social Origins of the Peasants' War of 1525 in Upper Swabia, in: Past \& Present 171 (1993), S. 30-65, hier S. 44f. 
reicht, aber bereits in Sichtweite ${ }^{16}$. Insofern ist es kein Zufall, daß der Bauernkrieg nicht nach Nord- und Ostdeutschland übergriff, obwohl sich v.a. im Osten viel weiterreichende Veränderungen als im Südwesten anbahnten, deren Gestalt bereits dreißig Jahre später als Gutsherrschaft bzw. zweite Leibeigenschaft in vollem Umfang zu erkennen sein sollte - jedenfalls in den Pioniergebieten ${ }^{17}$. Im Norden und teilweise auch im Osten waren jedoch wegen der höheren Verluste und der allgemein geringeren Bevölkerungsdichte auch in diesem Zeitraum oft noch ungenutzte Ressourcen vorhanden, durch die man die zusätzlichen Belastungen kompensieren konnte, indem z.B. Wüstungen von Bauern urbar gemacht bzw. weitläufig als Allmende genutzt wurden ${ }^{18}$.

Im Südwesten hingegen hatten sich die Bedingungen für grundherrschaftliche Offensiven, die an verschiedenen Stellen bereits im 15. Jahrhundert versucht worden, aber vielfach gescheitert waren, zu Beginn der Neuzeit spürbar verbessert. Die Zwölf Artikel der Bauern begegneten den neuen, erfolgversprechenden Anläufen der Herrschaftsträger mit einer gleichfalls neuen Position, die sich in der Formel vom "göttlichen Recht« ausdrückt. Meist wird sie mit guten Argumenten - als Radikalisierung bäuerlicher Positionen interpretiert, die bereits in den Bundschuhverschwörungen angeklungen sei und durch möglicherweise mißverstandene - Schlagworte aus dem Arsenal der frühen Reformation neue Nahrung erhalten habe ${ }^{19}$. Man könnte darin aber auch einen gewissen Eskapismus, den Ausdruck schwindenden Vertrauens in die eigene Verhandlungsmacht, erkennen. Allerdings spielt in den Artikeln neben der Berufung auf das »göttliche Recht" der Appell an die »Billigkeit» eine nahezu gleichwertige Rolle. Damit war der Verhandlungsweg, der sich in Oberschwaben und am Oberrhein im 15. Jahrhundert in zahlreichen Konfliktsituationen bewährt hatte ${ }^{20}$, wenigstens nicht prinzipiell ausgeschlossen.

${ }^{16}$ BliCKLE, Revolution (wie Anm. 1), S. 79f. Abweichend: SREENIVASAN, The Social Origins (wie Anm. 15), S. 44.

${ }_{17}$ Werner TROSSBACH, Einung, Willkür, Dorfordnung. Anmerkungen zur (Re-)Formierung dörflicher Gemeinden (13. bis 16. Jahrhundert), in: Jens FLEMMING u.a. (Hg.), Lesarten der Geschichte. Ländliche Ordnungen und Geschlechterverhältnisse. Festschrift für Heide Wunder zum 65. Geburtstag, Kassel 2004, S. 597-620.

${ }^{18}$ Gerhard HEITZ, Bäuerlicher Bodenbesitz und Wüstungsnutzung in Ruppin im 17. Jahrhundert, in: Probleme der Agrargeschichte des Feudalismus und des Kapitalismus 20 (1989), S. 21-26, hier S. 21, 24. Elisabeth SCHWARZE, Soziale Struktur und Besitzverhältnisse der ländlichen Bevölkerung Ostthüringens im 16. Jahrhundert, Weimar 1975, S. 110.

${ }^{19}$ BLICKLE, Revolution (wie Anm. 1), S. 145-149. Relativierend zum Bundschuh: Horst BUSzELlO, Joß Fritz und der Bundschuh za Lehen 1513, in: Peter BLICKLE, Thomas ADAM (Hg.), Bundschuh. Untergrombach 1502, das unruhige Reich und die Revolutionierbarkeit Europas, Stuttgart 2004, S. 80-121.

${ }^{20}$ Peter BIERBRAUER, Băuerliche Revolten im Alten Reich. Ein Forschungsbericht, in: Peter BLICKLE u.a., Aufruhr und Empörung. Studien zum bäuerlichen Widerstand im Alten Reich, München 1980, S. 1-68. 
Dieser pragmatischen Richtung entspricht, daß in den Zwölf Artikeln ein Gegenentwurf zur bestehenden Gesellschaft fehlt. Allerdings durchzieht die Zwölf Artikel - als Adjektiv oder Substantiv - ein polyvalentes, aber immer positiv aufgeladenes Schlüsselwort: Gemeinde. Indem sich die Zwölf Artikel nicht weiter über "Gemeinde« als Konzept verbreiteten, ließen sie an exponiertem Ort eine Leerstelle, die von den Rezipienten mit jeweils opportunen Inhalten gefüllt werden konnte. Die bäuerlichen Teilnehmer konnten die Idee "Gemeinde« auf ihren Erlebnishorizont beziehen, während sich die intellektuellen Verfasser von Programmentwürfen und Flugschriften »Gemeinde« mehr oder weniger als Basiselement eines künftigen Staatsaufbaus vorzustellen begannen. Damit stellt die - freilich nicht einheitlich interpretierte - Idee "Gemeinde« das Bindeglied der ländlich-bäuerlichen Beschwerden mit den intellektuellen Entwürfen dar, deren Ziel es oft auch war, die Orientierungen der Aufständischen an bereits existierende Reformentwürfe heran zu führen ${ }^{21}$.

Im Zuge des Aufstandes war "Gemeinde« weniger als Idee denn als Ensemble konkreter Ressourcen, Foren und Funktionen von Bedeutung. Die Glocken riefen die Gemeindemitglieder zusammen, Gemeindeversammlungen entschieden über die Teilnahme, die Inhaber von Gemeindeämtern übernahmen Führungsaufgaben. Im thüringischen Merxleben z.B. war einigen Bauern ein buche in die Hände gefallen, darinnen die 12 artigkel beschriben gewest. Sie versammelten die Gemeinde und das buch mußte ihnen George, der kirchner, als ein gemeiner knecht, ufm anger leßen ${ }^{22}$. Gemeindestrafen, z.B. der Ausschluß von Wasser und Weide oder die Errichtung eines Schandpfahls vor dem Haus, wurden gegen solche Gemeindemitglieder verhängt, die nicht am Aufstand teilnehmen wollten ${ }^{23}$.

$\mathrm{Da}$ der Begriff der Gemeinde in den bäuerlichen Beschwerdeschriften eine zentrale Stelle einnahm, lag jedoch nicht allein an den programmatischen Perspektiven und auch nicht nur an den organisatorischen Potenzen. Wichtiger als die Perspektiven, die der Idee »Gemeinde « inne wohnten, war für die Zwölf Artikel ebenso wie für die zahlreichen lokalen Beschwerdeschriften der Protest gegen Gefährdungen von als legitim empfundenen Positionen, die mit dem Begriff »Gemeinde« verbunden wurden ${ }^{24}$. Um Gründe und Begründungen dieses eher defensiven Protests zu verstehen, ist es sinnvoll, kurz auf die praktischen Funktionen einzugehen, die Gemeinden in deutschen Territorien am Vorabend des Bauernkrieges ausübten. Es war nicht anders als in vielen Teilen Europas. Gemeinden hatten seit dem 12. Jahrhundert einen Bereich

${ }^{21}$ Peter BlickLE, Der Bauernkrieg. Die Revolution des Gemeinen Mannes, München 1998, S. 90; DERS., Republiktheorie (wie Anm. 6), S. 196f.

${ }^{22}$ Walther Peter FuCHS (Hg.), Akten zur Geschichte des Bauernkriegs in Mitteldeutschland, Bd. 2, Jena.1942, ND Aalen 1964, S. 649.

${ }^{23}$ FRANZ, Bauernkrieg (wie Anm. 7), S. 115.

${ }^{24}$ BLICKLE, Revolution (wie Anm. 1), S. 72-77. 
definieren können, in dem sie in gewisser Weise Selbstverwaltung ausübten. Freilich war dieser Bereich nicht durch nationale oder regionale Gesetzgebung abgesteckt, eher schon durch Gewohnheitsrecht, das aber nicht statisch zu verstehen ist, sondern durch regionale und lokale Initiativen, teils auch konfliktträchtigen Zuschnitts, in wechselnden Kräfteverhältnissen unterschiedlich ausgestaltet wurde ${ }^{25}$.

Wenngleich die Gemeindefunktionen im einzelnen von Ort zu Ort differierten, kann doch verallgemeinemd festgehalten werden, daß der Umgang mit den kommunalen Ressourcen - die Nutzung wie die Sorge um die Substanzerhaltung - regionenübergreifend die wichtigste Aufgabe darstellte ${ }^{26}$. Der Kern der dörflichen Gemeinschaftsressourcen, der Allmendebereich, stammte meist aus der Verfügungsmasse von Villikationen und war zunächst in der Form von unklar definierten Nutzungsrechten zugänglich ${ }^{27}$. Im Zuge der Auflösung von Villikationen und vielfach auch im Kontext der Wüstungsepoche ${ }^{28}$ setzten sich insbesondere im Südwesten und in den Mittelgebirgen Eigentumsrechte der gestärkten Dorfgemeinden durch, nicht immer ohne langwierige Auseinandersetzungen mit Orts- und Landesherrschaften, die z.T. bis ins 18. Jahrhundert anhielten ${ }^{29}$.

Aus der Nutzung der kollektiven Ressourcen ergab sich eine Reihe von sekundären Entscheidungen: über Löhne und Preise, die Aufnahme oder Vergabe von Krediten oder die Verwendung der erzielten Gewinne ${ }^{30}$. Wurden die kollektiven Ressourcen periodisch an die Einzelhaushalte verteilt, zog auch dies eine Fülle weiterer Aufgaben nach sich, z.B. die Aufsicht über den Holzschlag im Gemeindewald und die Regelung der Beweidung, die neben der Ordnung der Flur genetisch als sursprünglicher Kernbereich ${ }^{31}$ dorfgemeindlicher Kompetenz erscheint. Verkaufserlöse, Pachtzahlungen, Strafen oder Gebühren - für die Nutzungen oder für die Aufnahme neuer Gemeindemit-

\footnotetext{
${ }^{25}$ TrossBaCH, Einung, Willkür, Dorfordnung (wie Anm. 17).

${ }^{26}$ Hartmut HARNISCH, Gemeindeeigentum und Gemeindefinanzen im Spätfeudalismus, in: Jahrbuch für Regionalgeschichte 8 (1981), S. 126-174.

${ }^{27}$ WARDE, Distribution of Resources (wie Anm. 9), S. 204; Hartmut ZüCKERT, Allmende und Allmendeaufhebung. Vergleichende Studien zum Spätmittelalter bis zu den Agrarreformen des 18./19. Jahrhunderts, Stuttgart 2003, S. 7.

${ }^{28}$ Hans JÄNICHEN, Beiträge zur Wirtschaftsgeschichte des schwäbischen Dorfes, Stuttgart 1970 , S. $168-178$.

${ }^{29}$ Albrecht STROBEL, Agrarverfassung im Übergang. Studien zur Agrargeschichte des badischen Breisgaus vom Beginn des 16. bis zum Ausgang des 18. Jahrhunderts, Freiburg i.Br. 1972, S. 177; ZÜCKERT, Allmende (wie Anm. 27), S. 233-239.

${ }^{30}$ Edwin Emst WEBER, Städtische Herrschaft und bäuerliche Untertanen in Alltag und Konflikt. Die Reichsstadt Rottweil und ihre Landschaft vom 30jährigen Krieg bis zur Mediatisienung, Bd. 1, Rottweil 1992, S. 288.

${ }^{31}$ Ibid., S. 220.
} 
glieder - wanderten in die Gemeindekasse ${ }^{32}$. Die damit verbundene Notwendigkeit einer wenigstens rudimentären Rechnungsführung nahm in dem Maße zu, wie sich Ausgaben und Einnahmen vor dem Hintergrund agrarischer und gewerblicher Konjunkturen - z.B. im Laufe des 16. Jahrhunderts ${ }^{33}$ - weiter summierten, wenn Gemeinden zusätzlichen Grundbesitz erwarben sowie Nutz- und Repräsentationsgebäude, Back-, Hirten- und Rathäuser übernahmen bzw. errichteten.

Beschlüsse von Gemeinden in Angelegenheiten, die sie weitgehend unabhängig regelten, wurden in hochmittelalterlichen Urkunden "Einung « bzw. "Willkür« genannt und seit dem späten 15. Jahrhundert in zahlreichen Dörfern - zunächst in einem breiten Gürtel vom Südwesten über Hessen und Franken bis nach Thüringen, Brandenburg und Preußen - in sogenannten Gemeindebriefen schriftlich fixiert ${ }^{34}$. Wenngleich es nur wenigen Gemeinden gelang, ihr Dorf- bzw. Einungsrecht völlig unabhängig zu formulieren, waren sie bei der praktischen Ausführung in weiten Bereichen ungestört, zumindest soweit die Grundherrschaft nicht in der Landwirtschaft engagiert war. In den meisten Fällen machten Orts- und Landesherrschaft lediglich Aufsichtsrechte geltend, die seit dem späten 15. Jahrhundert allerdings strenger gehandhabt wurden, z.B. auf dem wichtigen Gebiet der kommunalen Rechnungsfübrung ${ }^{35}$.

Seit dem 13. Jahrhundert begannen Mitglieder der bäuerlichen Gemeinde auch in der Ortskirche Funktionen auszuüben ${ }^{36}$. Noch weniger als für die Gemeindeverfassungen sind auf diesem Gebiet jedoch Verallgemeinerungen möglich. Während nahezu jedes Dorf eine Gemeinde ausbildete, gab es bis in die Moderne Pfarreien, die sich über mehrere Dörfer erstreckten, teils mit Filialkirchen in einzelnen Orten. Wo Dorf und Pfarrei nicht kongruent waren, versuchten seit dem Spätmittelalter insbesondere in größeren Dörfern die Gemeinden, Kirchen zu stiften bzw. Filial- zu Pfarrkirchen umzuwidmen und damit Dorf- und Pfarrgemeinde räumlich zur Deckung zu bringen. Im Kontext dieser Stiftungen rückte auch »die Pfarrerwahl in den Interessenkreis schwäbischer Bauern " $^{37}$, wenngleich ein förmliches Recht erst in den Zwölf Artikeln gefordert wurde, dann allerdings an erster Stelle.

Während die Befugnisse, die Gemeinden bei der Besetzung einer Pfarrstelle ausübten, schwer za verallgemeinern sind, läßt sich bezüglich der Verwaltung des Kirchengutes regionenübergreifend sagen, daß sie in den Händen von Dorf-

32 Gerlinde SCHLENKER, Die bäuerliche Gemeinde im Mittelelbe- und Saalegebiet vom 12. bis 15. Jahrhundert und ihr Verhältnis zum Grundherrn, in: Harz-Zeitschrift 48/49 (1998), S. 55-65, hier S. 58.

${ }^{33}$ HARNISCH, Gemeindeeigentum (wie Anm. 26), S. 143.

${ }^{34}$ TrossBaCH, Einung, Willkür, Dorfordnung (wie Anm. 17).

${ }^{35}$ Karl Siegfried BADER, Studien zur Rechtsgeschichte des mittelalterlichen Dorfes, Bd. 2:

Dorfgenossenschaft und Dorfgemeinde, Weimar 1962, S. 447f.

${ }^{36}$ Ibid., S. 202.

${ }^{37}$ Dietrich KURZE, Pfarrerwahlen im Mittelalter, Köln 1966, S. 254-258; Zitat S. 278. 
bewohnern lag. Bei der Bestellung der Kirchenpfleger, -väter oder -verwalter mußten Kirchengemeinde, Patronatsherren und Pfarrer zwar meist zusammen wirken $^{38}$, es gelang den dörflichen Verwaltern jedoch, einen erheblichen Dispositionsspielraum zu gewinnen, wenngleich sowohl (grund- oder orts-)herrschaftliche wie kirchliche Instanzen ein Überwachungs- oder Beteiligungstecht forderten. Angesichts eines gestärkten Selbstbewußtseins blieben Querelen der Heiligenpfleger oder Kastenverwalter mit den Pfarrern um Stiftungen und Geschenke nicht aus ${ }^{39}$. Nicht umstritten war die bereits in der alten Kirche verbreitete Praxis, Kirchenkapitalien als Kredite an Dorfbewohner auszuleihen ${ }^{40}$.

Problematischer als im Bereich des Einungsrechts und der Verwaltung des Kirchengutes wurden die Praktiken im Gerichtswesen bewertet. Bereits für das Hochmittelalter gilt: „Die Lokalisierung des Gerichts zwischen Herrschaft und Genossenschaft gehört zu den schwierigsten Problemen der Rechtsgeschichte $\aleph^{41}$. Im Spätmittelalter hatte sich an vielen Orten de facto das Problem vereinfacht, indem die Gemeinden die Schwäche der Grundherrschaft nutzten, Rechtsfindung und Verwaltung in eigene Hände nahmen und nach lokaler Observanz urteilten ${ }^{42}$. Die Gerichtsherrschaft war lediglich durch den SchultheiBen vertreten, der aber wiederum Mitglied der Gemeinde war. Vielleicht waren Verhältnisse verbreitet, wie sie noch im 17. Jahrhundert für ein Reliktgebiet etwas karikaturhaft beschrieben wurden: 1683 beklagte sich ein Beamter, daß die Einwohner des schwäbischen Ertingen

ihre schlaghändel und frävel nicht für die obrigkeit, sondern wellen alles vor ihrem groben bisselhirnigen paurengericht urtailen und aussprechen, auch darbei behaupten, das sy befuegt und berechtiget, alle gebotten und straffen ohnmaßgeblich der obrigkeit zue seten und zue annembsen, item auch wann ainer den andern wie über mit straichen tractiere und nur nit bluet gebe, seye solches nit höcher dann umb 5 bazen strafbar ${ }^{43}$.

Im Rheinland, wo die Gerichte grundherrschaftliche Register aufbewahrten, konnten Auseinandersetzungen um grundherrliche Rechte vor die bäuerlichen Schöffengerichte gezogen werden, sei es daß Grundherren miteinander, Bau-

${ }^{38}$ Sebastian SCHRÖCKER, Die Kirchenpflegschaft. Die Verwaltung des Niederkirchenvermögens durch Laien seit dem ausgehenden Mittelalter, Paderborn 1934, S. 133f.; BADER, Studien (wie Anm. 35), S. 208.

${ }^{39}$ SCHRÖCKER, Kirchenpflegschaft (wie Anm. 38), S. 64, 150, 152.

${ }^{40}$ Hans RÓSSLER, Pfarrer und Kirchpröbste. Die wirtschaftlichen Grundlagen der Pfarrseelsorge im 16. Jahrhundert, in: Zeitschrift für bayerische Landesgeschichte 64 (2001), S. 134-148.

${ }_{41}$ Peter BLICKLE, Kommunalismus. Skizzen einer gesellschaftlichen Organisationsform, Bd. 1: Oberdeutschland, München 2000, S. 32, 58.

${ }^{42}$ Heide WUNDER, Die bäuerliche Gemeinde in Deutschland, Göttingen 1986; BLICKLE, Kommunalismus (wie Anm. 41), S. 118.

${ }^{43}$ Zit. nach Peter BLICKLE, Die staatliche Funktion der Gemeinde und die politische Funktion des Bauern, in: DERS. (Hg.), Deutsche ländliche Rechtsquellen. Probleme und Wege der Weistumsforschung, Stuttgart 1977, S. 205-223, hier S. 216. 
ern untereinander oder Bauern mit Grundherren stritten. Man könnte regelrecht von einer unabhängigen Gewalt sprechen, mindestens konnten die Gerichte "Grundherren erheblich blockieren ${ }^{44}$.

Zahlreiche kleine Potentaten des deutschen Südwestens empfanden Gemeindebefugnisse dieser Art zunehmend als Hindernis für eine Steigerung der Abgaben und die Transformation ihrer Grundherrschaften in Gebilde mit nudimentär staatsähnlichem Anstrich. Die De-Facto-Kommunalisierung der Dorfgerichte hatte bereits im Spätmittelalter Gegenbewegungen ${ }^{45}$ ausgelöst. Äbte und Beamte versuchten durch die Einsetzung genehmer Richter, die Installation von territorialen Obergerichten, z.T. auch die Verfolgung mißliebiger Schöffen das Rad zurückzudrehen ${ }^{46}$. Außerdem wurden zunehmend herrschaftliche Eingriffe in das dörfliche Einungswesen beklagt ${ }^{47}$. Auch für diese spolitischen` Bereiche wäre von einer réaction seigneuriale zu sprechen.

Wer waren aber die Gemeinden, die den Arbeitsalltag ordneten, wichtige Ressourcen kontrollierten, in zahlreichen Gebieten Kirchenverwaltung und Gerichtsausübung an sich gebracht hatten, die im Bauernkrieg den Aufstand organisierten und in einzelnen Programmschriften als Fundament eines zukünftigen Staatsaufbaus vorgesehen waren? Seit langem wissen wir, daß Dörfer keine egalitären Gebilde darstellten, daß sie sogar soziale Unterschiede in geradezu krasser Ausprägung ausbilden konnten. Die dörfliche Realität war - freilich abhängig von den jeweiligen Erwerbsschwerpunkten - daher von oft extrem ungleichgewichtigen Formen der Kooperation, innerdörflicher Herrschaft und der Herausbildung bäuerlicher Eliten gekennzeichnet ${ }^{48}$.

Dem widerspricht deutlich das Bestreben, die sozialen Unterschiede aus der Gemeindeverfassung fernzuhalten, sie vielleicht sogar zu kompensieren, das seit dem 15. Jahrhundert die meisten Gemeindebriefe bzw. Dorfordnungen charakterisiert. In wichtigen Fällen verwiesen Gemeinden oft darauf, daß an der Entscheidungsfindung die ganze gemeind, reich und arm beteiligt gewesen sei $^{49}$. Die $" A r m e n \ll$ waren freilich nur insoweit einbezogen, als sie Land- und Hausbesitz hatten. Umgekehrt bedeutete dies, daß der Umfang des Landbesitzes in der Mehrzahl der Fälle zumindest de jure für die Teilhabe am sogenannten Dorfrecht keine Rolle ${ }^{50}$ spielte. Für die Land- und Hausbesitzlosen, deren Schicht im Verlauf des 16. Jahrhunderts zumindest regional stark zunahm,

44 Dieter SCHELER, Zur đörflichen Sozialstruktur am Niederrhein im späten Mittelalter, in: Tel Aviver Jahrbuch für deutsche Geschichte 22 (1993), S. 231-252, hier S. 239.

${ }^{45}$ STROBEL, Agrarverfassung (wie Anm. 29), S. 165.

${ }^{46}$ BLICKLE, Der Bauernkrieg (wie Anm. 21), S. 75-77.

${ }^{47}$ DERS., Kommunalismus (wie Anm. 41), S. 121.

${ }^{48}$ Werner Trossbach, Clemens ZimmermanN, Die Geschichte des Dorfes. Von den Anfängen im Frankenreich zur bundesdeutschen Gegenwart, Stuttgart 2006, S. 44, 69, 114, $164 f$.

${ }^{49}$ BADER, Studien (wie Anm. 35), S. 24-26, 150.

${ }^{50}$ Hermann GREES, Ländliche Unterschichten und ländliche Siedlung in Ostschwaben, Tübingen 1975, S. 17f,, 154-157. 
wurde hingegen in Anlehnung an städtische Verhältnisse der Beisitzerstatus entwickelt. Die meisten Dorfordnungen schlossen Beisitzerhaushalte von den Entscheidungsgremien, z.B. den Gemeindeversammlungen, aus ${ }^{51}$. Für den Allmendezugang gaben territoriale und lokale Regelungen den Ausschlag. In einigen Gemeinden Südwestdeutschlands wurden Beisitzerhaushalten Allmenderechte auf Widerruf oder gegen ein Weide- oder Holzgeld eingeräumt. Andere Gemeinden hielten sie explizit davon fern ${ }^{52}$.

Kleine Landbesitzer waren zwar an den Allmendenutzungen und den Entscheidungsprozessen formal beteiligt, mußten in der Realität aber erleben, daß Alltagsloyalitäten und das damit verbundene Machtgefälle oft in den politischen Raum - z.B. in die Gemeindeversammlung - hineinwirkten ${ }^{53}$. Eine ähnliche Schieflage fällt für die dörflichen Ämter, den eigentlichen Kristallisationspunkt der dörflichen Eliten, ins Auge. Freilich konnten sich bei der Besetzung von Ämtern, die stärker der Gemeinde verpflichtet und eher arbeitsaufwendig als prestigeträchtig waren, auch egalitäre Modi entfalten. Kollegialorgane - die Vierer, Achter oder Zwölfer - ermöglichten die Repräsentanz verschiedener Schichten des Dorfes ${ }^{54}$. In Jungingen bei Ulm mußte sich nach einer Ordnung von 1445 unter den Vierern jeweils mindestens ein Bauer und ein Seldner, ein Kleinstellenbesitzer also, befinden, in Bihlafingen bei Biberach war seit 1472 vorgeschrieben, daß das Gremium aus zwei Bauern und zwei Seldnem bestehen solle ${ }^{55}$. Utzmemmingen im Ries erließ 1651 folgende denkwürdige Bestimmung: Uff St. Georgitag sollen alle jahr zween vierer von den bauren auß den söldnern und von den söldnern 2 bauern zu vierern erwöhlt werden ${ }^{56}$.

Für das wichtigere Amt des Dorfvorstehers, das oft auch den Vorsitz im Dorfgericht beinhaltete, kamen dagegen meist die wohlhabenden Gemeindemitglieder in Frage. In Württemberg z.B. bildete ein regelrechtes Amtspatrizi$a^{57}$ durch Heiratsallianzen überlokale Netzwerke aus, deren Horizont sich dank politischer Partizipationsmöglichkeiten auf die Ebene von Amt und Land

${ }^{51}$ BADER, Studien (wie Anm. 35), S. 293, Anm. 125; Adolf THUMM, Die bäuerlichen und dörflichen Rechtsverhältnisse des Fürstentums Hohenlohe im 17. und 18. Jahrhundert, Benningen a. Neckar 1971, S. 140, 153.

${ }^{52}$ GREES, Unterschichten (wie Anm. 50), S. 157; Dietmar WEHRENBERG, Die wechselseitigen Beziehungen zwischen Allmendrechten und Gemeinfronverpflichtungen vornehmlich in Oberdeutschland, Stuttgart 1969, S. 58.

${ }^{53}$ THUMM, Rechtsverhältnisse (wie Anm. 51), S. 160; Bernd SCHILDT, Bauer - Gemeinde Nachbarschaft. Verfassung und Recht der Landgemeinde Thüringens in der frühen Neuzeit, Weimar 1996, S. 86.

${ }^{54}$ WUNDER, Gemeinde (wie Anm. 42), S. 96.

${ }^{55}$ GREES, Unterschichten (wie Anm. 50), S. 25; BLICKLE, Kommunalismus (wie Anm. 41), S. 52 .

${ }^{56}$ WeHRENBERG, Beziehungen (wie Anm. 52), S. 115.

${ }^{57}$ Karl Siegfried BADER, Dorfpatriziate, in: Zeitschrift für die Geschichte des Oberrheins 101 (1953), S. 269-274. 
ausdehnte. Einzelnachrichten aus dem 15. Jahrhundert ${ }^{58}$ stellen dar, wie in anderen schwäbischen Territorien führende Familien Dorfämter monopolisierten, zu ihrem Privatinteresse ausnutzten und andere Dorfbewohner regelrecht terrorisierten. Auch die kirchlichen Amtsträger stammten sozial meist aus den Oberschichten, oft waren sie in Personalunion Inhaber von profanen Ämtern ${ }^{59}$. Welches Selbstbewußtsein mit einem kirchlichen Amt verbunden war, zeigt sich z.B. darin, daß im thüringischen Schleid die Heiligenmeister Hans Wilk und Cort Egkart 1475 quasi als Bauherren ihre Namen kunstvoll in ein neu errichtetes gotisches Taufbecken einmeißeln ließen ${ }^{60}$.

Wenn der Bauernkrieg als Gemeinderevolte bezeichnet wird, schließt sich somit die Frage an, inwieweit sich die Schieflagen, die diese Organisationsform beinhalten konnte, in der Aufstandsbewegung reproduzierten. Der Neuinterpretation des Bauernkrieges, die David Sabean bereits vor längerem auf der Basis oberschwäbischer Quellen vorgelegt hat ${ }^{61}$, liegt genau diese Überlegung zugrunde, sie geht aber noch einen Schritt weiter. Sabean stellt nicht die Gegensätze zwischen Bauern und Obrigkeiten in den Mittelpunkt seiner Betrachtung, sondern die Konflikte innerhalb der Dörfer, zwischen Großbauern und Kleinstellenbesitzern bzw. Unterschichten. Sie drehten sich um die Kontrolle der kommunalen Ressourcen, in Oberschwaben z.B. um die Forderung der Unterschichten nach partieller Aufteilung der Allmende. Neuere Forschungen haben ähnliche Gegensätze auch in anderen Gebieten dokumentieren können ${ }^{62}$.

Die seit langem bekannte Tatsache ${ }^{63}$, daß die bäuerlichen Oberschichten unter den Führungspersonen im Bauernkrieg stark überrepräsentiert waren, gewinnt damit neue Brisanz. Lokale Autonomie im Einungsrecht, insbesondere bei der Kontrolle über die Allmende, Gerichtsausübung durch bäuerliche Schöffen, Pfarrerwahl durch die Gemeinde, Autonomie bei der Besetzung dörflicher Ämter - vor dem Hintergrund der Verwaltungspraxis und der Aufstandsorganisation erscheinen diese Forderungen als Kernprogramm einer kleinen Oberschicht, eines bäuerlichen Kreises im engeren Sinne des Wortes, einer Art Zwischenschicht der lokalen Gesellschaften, die zwar fest im Dorf verankert, jedoch auch mit Aufgaben befaßt war, die als >Herrschaft bezeichnet werden können. Die Angehörigen dieser Schicht sahen ihre Positionen durch eine réaction seigneuriale bedroht, die dadurch ein unkluges Element enthielt, daß sie nicht nur auf die materiellen, sondern auch auf die politischen

\footnotetext{
${ }^{58}$ Casimir BUMILLER, Studien zur Sozialgeschichte der Grafschaft Zollern im Spätmittelalter, Sigmaringen 1990, S. 231, Anm. 123.

${ }^{59}$ SREENIVASAN, The Social Origins (wie Anm. 15), S. 39.

${ }^{60}$ Erwin STURM, Bau- und Kunstdenkmale in Schleid, in: Buchenblätter (Beilage zur Fuldaer Zeitung) 70 (März 1997), S. 27.

${ }^{61}$ SABEAN, Landbesitz (wie Anm. 12).

${ }^{62}$ SREenIVASAN, The Social Origins (wie Anm. 15), S. 32f.

${ }^{63}$ FRANZ, Die Führer im Bauernkrieg (wie Anm. 2).
} 
Besitzstände dieser Schicht zielte. Wenn dieser Kreis jedoch Gemeinderechte gefährdet sah, hatte er eine andere Grundlage im Auge als die Masse der Kleinstellenbesitzer.

Auch situativ kann sich diese Einschätzung auf zahlreiche Indizien beziehen. Wenn es z.B. heißt, »die Gemeinde " habe für ein Dorf die Teilnahme am Aufstand beschlossen, dann konnte das so aussehen, daß die in der Gemeinde ohnehin führenden Vertreter auch im Bauernkrieg mehr oder weniger eigenmächtig die Teilnahme am Aufstand beschlossen. Kleinstellenbesitzer und Landlose wurden entweder nicht informiert oder es blieb ihnen nichts anderes übrig, als sich anzuschließen, oft ohne zu wissen, um was es konkret ging ${ }^{64}$, ähnlich wie in der Grande Peur der Französischen Revolution. Allerdings wissen wir nicht, in welchem Ausmaß dies für die Gesamtbewegung der Fall war, und selbst auf regionaler Ebene liegen nur Einzelnachrichten vor.

Waren die Erhebungen im Bauernkrieg also »nach außen gestülpte Konflikte um die angemessene Verteilung der vorhandenen Ressourcen im Dorf ${ }^{65}{ }^{\text {? }}$ ? Es klingt wenig logisch, daß Großbauern einen regionenübergreifenden Krieg gegen Adel und Klerus vom Zaun brachen, um ihre Dominanz vor Ort - z.B. die Kontrolle der Allmende gegen die Bestrebungen ihrer ärmeren Nachbarn zu bewahren. Dafür hätte man andere Mittel einsetzen können, möglicherweise solche, die auf dem Schulterschluß mit der Grundherrschaft beruhten. An Plausibilität gewinnt die Sabean-These jedoch dann, wenn sie etwas zurückhaltender formuliert wird. So ist zu diskutieren, ob es den dörflichen Oberschichten gelungen ist, die Gemeinde für ihre partikularen Interessen zu mobilisieren ${ }^{66}$.

Dazu konnten sie einmal den Vorsprung nutzen, der auf der Beherrschung der dörflichen Institutionen beruhte, zum anderen auch ihre Macht über die privaten Ressourcen des Dorfes, v.a. über den Arbeitsmarkt und den Markt für Pachtgrundstücke ${ }^{67}$. Leider schweigen die Quellen der Bauernkriegszeit, wie diese Macht furr die Mobilisierung der Unterschichten eingesetzt wurde, was auch daran liegen kann, daß oft schon der stumme Zwang der Verhältnisse die Kleinbesitzer zur Willfährigkeit bewogen haben mag. Andererseits: Auch Kleinbesitzer waren - wie der vielfältige innerdörfliche Streit um Allmendenutzungen zeigt ${ }^{68}$ - durchaus konfliktfähig und unterlagen keinesfalls immer der Übermächtigung durch die Reichen im Dorf. Die berühmte Zimmer'sche Chronik enthüllt allerdings weitere Hebel, die von den dörflichen Eliten hätten bewegt werden können. So sei befunden worden, daß der größte Teil der Tage-

${ }^{64}$ Roy L. VICE, The Leadership and Structure of the Tauber Band during the Peasants' War in Franconia, in: Central European History 21 (1988), S. 175-195, hier S. 179.

${ }^{65}$ So die Interpretation der Sabean-These bei BLICKLE, Der Bauernkrieg (wie Anm. 21), S. 125 .

${ }^{66}$ SREENIVASAN, The Social Origins (wie Anm. 15), S. 39.

${ }^{67}$ Trossbach, ZimmermanN, Geschichte des Dorfes (wie Anm. 48), S. 155-158.

${ }^{68}$ WARDE, Distribution of Resources (wie Anm. 9); STROBEL, Agrarverfassung (wie Anm. 29), S. 172 . 
löhner der mair sone, dochtermener oder nechste verwandte gewesen ${ }^{69}$. Freilich wird auch für diese Bindungen - Blutsverwandtschaft und Schwägerschaft - aus den Quellen kaum deutlich, wie sie konkret eingesetzt wurden. An sich besaßen sie ein Janusgesicht; es handelte sich um "zweischneidige ${ }^{70}$ Verhältnisse, die die dörfliche Machtausübung der Großbauern nicht nur verstärken, sondern auch relativieren konnten.

$\mathrm{Da}$ die Wirksamkeit dieser Kräfte für die Bauernkriegszeit kaum zu fassen ist, muß zunächst mit einer Analyse der Interessen vorlieb genommen werden. Aber auch auf diesem Weg kann man der Antwort auf die Frage näher kommen, ob die Gemeinden im Bauernkrieg in erster Linie Partikularinteressen der bäuerlichen Oberschichten verfochten. Dabei kann nicht ignoriert werden, $\mathrm{da} ß$ es innerhalb eines Dorfes nicht nur gegensätzliche Interessen gab. Von Steuern, Fronen und Allmendebeschränkungen durch die Herrschaft waren alle landbesitzenden Schichten im Dorf betroffen, wenn auch nicht immer im gleichen Maße. Von einer Politik, die - wie in Oberschwaben - auf die Änderung der Besitzqualitäten zielte und die Dispositionsfreiheit der großbäuerlichen Lehensinhaber einschränkte, waren nicht allein diese, sondern auch deren "Söhne, Tochtermänner und nächste Verwandte« betroffen. Ein Verbot der Einrichtung von Häuslingsstellen ${ }^{71}$, wie es im Herrschaftsbereich des Klosters Weißenau seit etwa 1480 ausgesprochen wurde, betraf z.B. sowohl die künftigen Häuslinge bzw. Inwohner wie die künftigen Hoferben und nicht zuletzt die besitzenden Eltern, die beiden Teilen gerecht zu werden hatten.

Vor diesem Hintergrund ist es kein Zufall, daß die Auseinandersetzungen im Bauernkrieg oft in solchen Gebieten ein besonders heftiges Ausmaß erreichten, wo die Grundherrschaften gegen den Willen von Eltern und Kindern, erbenden und weichenden Geschwistern, Bauern und Unterschichten Teilungen oder andere interne Abfindungsregelungen blockierten. Ähnlich wie das Kloster Weißenau, von dem noch die Rede sein wird, wurde z.B. auch das Stift Backnang, das weichende Geschwister zu landloser Existenz gezwungen hatte, im Bauernkrieg geplündert ${ }^{72}$, während in den Dörfern des benachbarten schwäbischen Strohgäus, wo die Grundherrschaft Neugründungen und Teilungen von Bauernstellen begünstigte, »keine Aktivitäten feststellbar sind«. Für eine Kleinregion des Nordschwarzwaldes ist gleichfalls beobachtet worden, daß sich eher Dörfer mit unausgewogener Sozialstruktur am Aufstand beteiligten ${ }^{73}$.

\footnotetext{
${ }^{69}$ SABEAN, Landbesitz (wie Anm. 12), S. 101.

${ }^{70}$ DERS., Das zweischneidige Schwert. Herrschaft und Widerspruch im Württemberg der frühen Neuzeit, Frankfurt a.M. 1990.

7 DERS., Landbesitz (wie Anm. 12), S. 46.

${ }^{72}$ GEYER, Backnang (wie Anm. 12), S. 293.

${ }^{73}$ Kerstin ARNOLD, Bauernleben und Herrschaftsstreben. Sozialgefuige, wirtschaftliche Verhältnisse und Herrschaftsstrukturen im Nordschwarzwald (8.-16. Jahrhundert), Tübingen 2002, S. $218 f$.
} 
Auch in anderen Territorien standen die Obrigkeiten dem Breitenwachstum der Unterschicht ablehnend oder zumindest hilf- und konzeptionslos gegenüber. Im Gebiet der Reichsstadt Ulm suchte man bereits zu Beginn der Neuzeit die Zunahme ihrer Zahl durch Hausbauverbote einzudämmen. In ähnlicher Weise reagierte die Abtei Ottobeuren, allerdings erst Mitte des 16. Jahrhunderts. Die weitere Expansion unterbäuerlicher Haushalte konnte dadurch jedoch nur begrenzt verhindert werden. In Ottobeuren ging man zur Teilung von Häusern über ${ }^{74}$, im Ulmer Umland entstanden land- und hausbesitzlose Haushalte, die auf textilwirtschaftlichem Erwerb beruhten ${ }^{75}$. Dies hatte den Nebeneffekt, daß diese Haushalte zumindest auf der Schiene des Besitzes nicht mehr mit der Grundherrschaft verbunden waren, da sie weder Land noch Haus besaßen.

Auch wo keine administrativen Einschränkungen für die Bildung unterbäuerlicher Haushalte bestanden, war in vielen Fällen nicht die Grundherrschaft der wichtigste Ansprechpartner der neu zu gründenden Haushalte, auch dann nicht, wenn es sich nicht um gänzlich land- und hausbesitzlose Bewohner handelte. Bereits im 13. Jahrhundert häufen sich die Indizien, daß ein größer werdender Teil der Behausungen nicht mehr auf »grundherrschaftlichem Boden« stand ${ }^{76}$. Dagegen finden sich zahlreiche Angaben, daß bäuerliche Hufner die Errichtung von Häusern und Hütten auf ihrem Grund und Boden gestatteten, was seinen sprachlichen Ausdruck im - freilich mehrdeutigen - Begriff des Hintersassen bzw. Hintersettlers fand ${ }^{77}$. Moderne geographische Arbeiten sprechen von Nachsiedlerschichten. Einblicke in den Vorgang der Nachsiedlung gestattet ein Abgabenverzeichnis für Buchdorf bei Donauwörth. Dort lag hinter einer bäuerlichen Hofstatt ein Acker, von dem es 1319 heißt: In parte eiusdem agri sunt constructe due selde ${ }^{78}$. Oft standen die bereits angesprochenen verwandtschaftlichen Bindungen im Hintergnund. In einem Nachtrag des Lagerbuches von 1526 wird der Bau eines Hauses in Sonderbuch bei Ulm folgendermaßen begründet: Als $H$. J., Inwohner zu S., zu kainem underschlauf kommen können, hat ime sein bruoder, L. J. daselbsten, ain hauslin in seinem garten, der in sein hoffguett gehörig und der kellerey Blaubeuren zinsbar etc., $z u$ bawen, uff unsers gnedigsten füsten und herrn etc. ratification vergund $t^{79}$.

Die Salbücher Hessens und der benachbarten Kleinterritorien reflektieren die Beteiligung einer anderen Kraft, der Gemeinden. Einen Einblick in die damit verbundene Dynamik ermöglicht das Beispiel von Braach bei Roten-

\footnotetext{
${ }^{74}$ SREENIVASAN, Ottobeuren (wie Anm. 12), S. 165-172.

${ }^{75}$ Hermann GREES, Die bäuerliche Kulturlandschaft der Ostalb, Diss. (Masch.) Tübingen 1961, S. 269-274.

${ }^{76}$ Eckhart SCHREMMER, Die Wirtschaft Bayerns. Vom hohen Mittelalter bis zum Beginn der Industrialisierung, München 1970, S. 112; GREES, Unterschichten (wie Anm. 50), S. 89.

${ }^{77}$ Ibid., S. 105, 118-120; SCHWARZE, Soziale Struktur (wie Anm. 18), S. 76.

${ }^{78}$ GREES, Unterschichten (wie Anm. 50), S. 121.

${ }^{79}$ Tbid., S. 122f., Anm. 805.
} 
burg. Das Dorf bestand 1538 aus 35 Häusern, darunter waren bereits sieben sogenannte Anbauern auf der Gemeinde angesiedelt. 1579 umfaßte das Dorf 65 Häuser, 22 standen auf der Gemeinde ${ }^{80}$. Auch in württembergischen, bayerischen und thüringischen Dörfern wurden auf Gemeindegrund neue Wohnstätten errichtet ${ }^{81}$. Bauland konnte auch von kirchlichen Institutionen zur Verfügung gestellt werden. Der Landerwerb wurde von den gleichen Kräften erleichtert, die auch Bauplätze zur Verfügung gestellt hatten. Wurde eine Selde auf bäuerlichem Grund erbaut, gab der Bauer vielleicht auch etwas Land dazu ${ }^{82}$. Auch die Ortskirche wurde aktiv. Noch im 18. Jahrhundert entrichteten in württembergischen, ostschwäbischen und in bayerischen Dörfern zahlreiche Selden »dem Heiligen « Grundzinsen ${ }^{83}$. Im Südwesten gestatteten bisweilen Kredite aus dem örtlichen Kirchenfonds die Anschaffung einer Kuh, "worin sich die 'soziale Bedeutung solcher örtlicher Geldgeber erweist ${ }^{84}{ }^{24}$. Die Ausstattung von ärmeren Dorfbewohnern mit Bauplätzen und Land durch einzelne Bauern bzw. durch Ortskirchen und Gemeinden, durch Institutionen also, die von Bauern dominiert wurden, bildete aber auch eine zusätzliche Basis von Patronage- und damit von Machtverhältnissen.

Gemeindeinstitutionen traten hinsichtlich der Landausstattung auch bei teils spektakulären Rodevorgängen in Erscheinung, die auf die Rechte der Grundherrschaft wenig Rücksicht nahmen. Um 1550 stellte der mit der Besichtigung der dörflichen Strukturen in der Grafschaft Ziegenhain betraute hessische Schultheiß fest, daß sich die Verhältnisse in den vorangegangen hundert Jahren erheblich verändert hatten, und zwar nicht allein durch Güterteilungen und die Unterschlagung von Zinsen, sondern vor allem durch umfangreiche Rodungen, für die z.T. kein Zins entrichtet werde ${ }^{85}$. Durch die Anlage von Salbüchern sollten die Rodungen, die bereits Ende des 15. Jahrhunderts eingesetzt hatten, seitens der Landesherrschaft legalisiert, die Grundstücke aber auch mit Zinsen belastet werden.

Für die dörflichen Konstellationen zeigt der Ablauf einzelner Rodungen, $\mathrm{da} \beta$ es auch innerhalb von asymmetrischen Strukturen Spielräume für die Aushandlung divergierender Interessen gab, daß sich die Abhängigkeiten in

\footnotetext{
${ }^{80}$ Helmut JÄGER, Entwicklungsperioden agrarer Siedlungsgebiete im mittleren Westdeutschland seit dem frühen 13. Jahrhundert, Würzburg 1958, S. 91.

${ }^{81}$ Theodor KNAPP, Neue Beiträge zur Rechts- und Wirtschaftsgeschichte des wurttembergischen Bauernstandes, Tübingen 1919, ND Aalen 1964, S. 96; SCHWARZE, Soziale Struktur (wie Anm. 18), S. 72, 86.

${ }^{82}$ GREES, Unterschichten (wie Anm. 50), S. 121.

${ }^{83}$ KNAPP, Neuere Beiträge (wie Anm. 81), S. 96; GrEES, Unterschichten (wie Anm. 50), S. 115.

${ }^{84}$ Ibid., S. 121, 136.

${ }^{85}$ Martin BORN, Wandlung und Beharrung ländlicher Siedlung und Wirtschaft. Untersuchungen zur frühneuzeitlichen Kulturlandschaftsgenese im Schwalmgebiet, Marburg 1961, S. 27, 32 .
} 
den Dörfern jedenfalls nicht immer in strikter Dominanz der Großbauern ausdrückten. Im hessischen Balhorn gingen die großen Bauern zwar überproportional begünstigt aus den Rodungen des 16. Jahrhunderts hervor ${ }^{86}$. In anderen Teilen Hessens profitierten allerdings Kleinbauern weitaus am meisten - wie auch im schaumburgischen Dorf Lindhorst ${ }^{87}$. Auch partielle Aufteilungen der Allmende waren möglich - anders als offenbar 1525 in Oberschwaben. Im ostschwäbischen Gussenstadt wurden bei einer solchen Aktion in der zweiten Hälfte des 16. Jahrhunderts alle Haushalte gleichmäßig berücksichtigt ${ }^{88}$. In Loshausen in der Schwalm wurde Gemeindeland zu gleichen Teilen unter 36 Nutzungsberechtigten ausgegeben ${ }^{89}$, wobei allerdings $z u$ fragen ist, ob auch Landlose bedacht wurden.

In Bayern schließlich war es in einigen Ämtern vor dem Hintergrund eines starken Bevölkerungsdrucks im späten 16. Jahrhundert $»$ zu einer intensiven Binnenkolonisation in den Mooren und Moosen « gekommen, die von den Kleinstellenbesitzern in eigener Initiative durchgeführt wurde. Die Familien, die sich der Mühe unterzogen hatten, auf diesen Flächen zu siedeln, waren bis ins 18. Jahrhundert "vor grundherrschaftlichem Zugriff sicher $"{ }^{90}$. Wenngleich quantitative Befunde ausstehen, kann festgehalten werden, daß es eine unbekannte, aber durchaus stattliche Zahl von Unterschichtenhaushalten gab, die mit einer Grundherrschaft nicht mehr durch Landbesitz verbunden waren, sei es, daß sie kein Land hatten, sei es, daß sie ihren kärglichen Haus- und Landbesitz anderen Quellen verdankten.

Wenn sie dennoch in einen Herrschaftszusammenhang integriert waren, geschah dies zumindest im Südwesten offenbar durch einen Rechtstitel, gegen den sich wie gegen keine andere Institution die prinzipielle Ablehnung der Bauernkriegsteilnehmer richtete, auch in einem gemäßigten Dokument wie den Zwölf Artikeln: die Leibeigenschaft ${ }^{91}$. Dieser Rechtstitel war im Horizont der Aufgabe der Villikationsverfassung entstanden. Er konnte verschiedenartig ausgestaltet werden und entsprach verschiedenen Bedürfnissen. So hatte er ein rückwärts-, aber auch ein vorwärtsgewandtes Gesicht. Zum einen diente er dazu, Villikationen als Personenverbände zusammenzuhalten, obwohl sie nach

${ }^{86}$ Harald KRIEGER, Balhorn. Ein Dorf auf dem Weg aus dem Mittelalter, Frankfurt a. d. Oder 1998, S. 62.

${ }^{87}$ BORN, Wandlung (wie Anm. 85), S. 36; Hans Werner RoTHE, Zur Geschichte der lăndlichen Gesellschaft im Schaumburger Land: Lindhorst, o.O. 1998, S. 302.

${ }^{88}$ Bertram FINK, Die Böhmenkircher Bauernrevolte 1580-1582/83, Leinfelden-Echterdingen 2004, S. 68.

${ }^{89}$ BORN, Wandlung (wie Anm. 85), S. 33.

${ }^{90}$ Rudolf SCHLOGL, Zwischen Krieg und Krise. Situation und Entwicklung der bayerischen Bauernwirtschaft im 17. Jahrhundert, in: Zeitschrift fur Agrargeschichte und Agrarsoziologie 40 (1992), S. 133-167, hier S. 136.

${ }^{91}$ BLICKLE, Revolution (wie Anm. 1), S. 40-50; DERS., Von der Leibeigenschaft zu den Menschenrechten. Eine Geschichte der Freiheit in Deutschland, München 2003. 
der Aufgabe der Eigenwirtschaften keine Funktion mehr hatten und v.a. bei verstreuter Lage kaum noch zu übersehen waren. Das Verbot der Heirat außerhalb des Leibeigenschaftsverbandes und Mobilitätsbeschränkungen sollten vor dem Hintergrund der spätmittelalterlichen Agrarkrise die Abwanderung in Städte oder in andere Herrschaften erschweren und v.a. eine Entfremdung grundherrschaftlich gebundenen Landes verhindern. Zum anderen diente der Rechtstitel einem smodernen ‘ Anliegen, der Umwandlung von Villikationen in vorstaatliche Gebilde, die von der Leibeigenschaft als einer Vorform der Staatsbürgerschaft zusammengehalten werden sollten, wobei der Widerspruch zwischen villikaler Streulage und dem Territorialprinzip der modernen Staaten durch Leibeigenenaustausch gelöst werden konnte.

Die bäuerlichen Oberschichten waren v.a. durch die Heiratsverbote betroffen, die massiv in familiäre Strategien eingriffen. Übertretungen wurden mit Entzug von Land bestraft ${ }^{92}$. Auch auf andere Weise - v.a. durch Ansprüche auf das Erbe - wurde versucht, auf den Besitz der Leibeigenen zuzugreifen, um grundherrschaftliche Dominanz $\mathrm{zu}$ festigen oder wiederherzustellen. Im 15. Jahrhundert gelang es den Betroffenen in einer Reihe von Verhandlungen in zahlreichen süddeutschen Herrschaften allerdings, die Erbschaftsansprüche der Leibherren vom Landbesitz weg und hin auf Vieh- und Mobilienbesitz zu lenken. Meist wurden die Ansprüche auf den Fall beschränkt, daß ein Haushaltsvorstand starb, dergestalt daß das beste Kleid oder das beste Stück Vieh (Todfall bzw. Besthaupt) an den Leibherrn fiel. Manchmal fanden sich auch prozentuale Beschränkungen ${ }^{93}$. Oft wurden die Verträge von den Obrigkeiten aber nicht eingehalten.

Trotz gewisser Erfolge im Vorfeld vereinigte die Ablehnung der Leibeigenschaft alle am Aufstand beteiligten Schichten in einem Maße, das geeignet war, interne Interessengegensätze zurücktreten zu lassen. Das Gefühl der Diskriminierung durch die bloße Bezeichnung stellte sich nicht erst in der Aufklärungszeit ein, sondern durchzieht auch die Dokumente des Bauernkrieges ${ }^{94}$. Materiell waren von den Todfallabgaben die Unterschichten überproportional betroffen: Das beste Stück Vieh, das beim Tod des Haushaltsvorstandes gefordert wurde, war bei ihnen oft das einzige. Das impliziert, daß in den vertraglichen Regelungen des 15. Jahrhunderts, die den Erbschaftsanspruch der Grundherrschaft vom Land- auf den Viehbesitz verschoben hatten, vielfach einseitig besitzbäuerliches Interesse zum Ausdruck kommt ${ }^{95}$. Der bäuerliche

\footnotetext{
92 UlBRICH, Leibherrschaft (wie Anm. 12), S. 111, 149, 261.

${ }^{93}$ Ibid., S. 66, 268f.; SABEAN, Landbesitz (wie Anm. 12), S. 42f.

${ }^{94}$ BLICKLE, Der Bauernkrieg (wie Anm. 21), S. 55, 63-69; ULBRICH, Leibherrschaft (wie Anm. 12), S. 302; SReEnIVASAN, The Social Origins (wie Anm. 15), S. 47.

95 SABEAN, Landbesitz (wie Anm. 12), S. 42f. Manchmal konnte nur ein halbes Stùck verlangt werden, da sich zwei Haushalte offenbar ein Tier teilten: ULBRICH, Leibherrschaft (wie Anm. 12), S. 304f. Dagegen steht für Ottobeuren die Beobachtung von SREENIVASAN, The
} 
Landbesitz war von den Übergriffen der Leibherrschaft weitgehend geschützt, was insbesondere landlosen Unterschichten wenig nutzte, wohingegen das Vieh, das diese auf der Allmende oder auf Pachtland halten konnten, den Zugriffen weiterhin ausgeliefert war. Auch der sogenannte kleine Zehnt, der nicht mit der Leibeigenschaft in Verbindung stand, belastete als Blutzehnt die meist auf Tierhaltung basierende Wirtschaftsweise ${ }^{96}$ der Landlosen und Landarmen stärker als die der Großbauern, die eher auf den Ackerbau setzten. Als Zehnt, der für Brachfrüchte wie Gemüse und Faserpflanzen erhoben wurde, benachteiligte er außerdem Betriebe, die im Umkreis der Städte diese Marktprodukte anbauten, darunter in der Mehrzahl Kleinstellen ${ }^{97}$.

Wurden Mobilitätsbeschränkungen aus der Leibeigenschaft abgeleitet, behinderten sie nicht allein großbäuerliche Heiratsstrategien, sie trafen auch bei den Unterschichten einen Nerv ${ }^{98}$. Landlose und Kleinstellenbesitzer verdienten ihren Lebensunterhalt durch eine Vielfalt von Aktivitäten, die ihnen äußerste Flexibilität abverlangten. V.a. in Weinbaugebieten waren zahlreiche Wanderarbeiterinnen und Wanderarbeiter tätig, die teils in einer Stadt wohnten und auf dem Dorf arbeiteten oder auch umgekehrt ${ }^{99}$. Auch in Getreideanbaugebieten war in der Erntezeit Wanderarbeit an der Tagesordnung ${ }^{100}$. Andere ländliche Lohnarbeiter wiederum pendelten täglich zwischen ihrem ländlichen Wohnsitz und einem städtischen Arbeitsplatz ${ }^{101}$ oder einer ländlichen Arbeitsgelegenheit in einem anderen Dorf ${ }^{102}$.

Ähnlich eng waren die Verbindungen zwischen Stadt und Land, wenn sie auf der arbeitsteiligen Herstellung gewerblicher Produkte beruhten. Im Memminger Umland hatte die Leder- und Sensenherstellung zu Beginn der Neuzeit ländliche Filialen, außerdem lieferten dörfliche Wagner fertige Holzräder, die in der Stadt beschlagen wurden ${ }^{103}$. Die Herstellung von Fässern in Weinbau-

Social Origins (wie Anm. 15), S. 41, daß Unterschichten oft nicht in die Leibeigenschaft, zumindest nicht in die des Klosters, einbezogen waren.

${ }^{96}$ DERS., Ottobeuren (wie Anm. 12), S. 153, 198.

${ }^{97}$ STROBEL, Agrarverfassung (wie Anm. 29), S. 140.

${ }^{98}$ ULBRICH, Leibherrschaft (wie Anm. 12), S. 306.

${ }^{99}$ Roy L. VICE, Vineyards, Vinedressers and the Peasant's War in Franconia, in: Archiv fur Reformationsgeschichte 79 (1988), S. 138-157.

${ }^{100}$ Der Komplex ist im Gegensatz zu Frankreich nicht systematisch erforscht. Hinweise: Karl-Sigismund KRAMER, Volksleben im Fürstentum Ansbach und seinen Nachbargebieten (1500-1800), Würzburg 1961, S. 244f.

${ }^{101}$ Leipzig: Uwe SCHIRMER, Das Amt Grimma 1485 bis 1548. Demographische, wirtschaftliche und soziale Verhältnisse in einem kursächsischen Amt am Ende des Mittelalters und zu Beginn der Neuzeit, Beucha 1996, S. 68.

${ }_{102}$ Thomas ROBISHEAUX, Rural Society and the Search for Order in Early Modern Germany, Cambridge 1989, S. 74f., 80.

${ }^{103}$ Rolf KIESSLING, Oberschwaben - eine offene Gewerbelandschaft, in: BLICKLE, Oberschwaben (wie Anm. 6), S. 27-55, hier S. 48. 
gebieten war gleichfalls auf Stadt und Land verteilt ${ }^{104}$. Auf freien Zugang $\mathrm{zu}$ Märkten und Verteilungszentren waren die unterbäuerlichen Schichten nicht zuletzt in den Gebieten der textilen Protoindustrie angewiesen. In Augsburg und den größeren Siedlungen des Umlandes z.B. verkaufte die Landbevölkerung regelmäßig Flachs und Garn auf den Märkten ${ }^{105}$, außerdem lieferten Spinnerinnen und Spinner vom Lande im Wochenturnus fertiges Baumwollgarn bei den größeren Webern ab und nahmen Spinnmaterial mit nach Hau$\mathrm{se}^{106}$. Beschränkungen waren nicht nur lästig, sie konnten sich auch existenzgefährdend auswirken. Die weitgehende, wenn auch nicht totale Kongruenz von Bauernkriegsgebieten und Regionen mit hoher Städtedichte bzw. landwirtschaftlicher Spezialisierung oder gewerblicher Ausrichtung kann vor diesem Hintergrund nicht als Zufall erscheinen.

Es gab also Interessengegensätze zwischen Großbauern und Obrigkeiten und solche zwischen Bauern und unterbäuerlichen Schichten in den einzelnen Gemeinden. Daneben dürfen aber die gemeinsamen Interessen von Bauern und Unterschichten nicht vergessen werden, die sie gegen die Obrigkeiten verbanden, und v.a. nicht die Interessengegensätze, die zwischen den Unterbäuerlichen und den Herrschaftsträgern bestanden. Die Zwölf Artikel trugen diesen Besonderheiten durchaus Rechnung. Neben der Aufgabe der Leibeigenschaft wurde in einem zusätzlichen Artikel die Abschaffung des Todfalls verlangt, was insofern keine Dopplung war, da oft der Todfall auch dann verlangt wurde, wenn keine förmliche Leibeigenschaft bestand ${ }^{107}$. Ebenso eindeutig wurde der Verzicht auf den "kleinen Zehnten“ gefordert. Das Hauptdokument des Bauernkrieges drückte also keineswegs allein die Interessen der Großbauern aus, sondern enthielt - wie auf den Titelseiten von einigen der zahlreichen Drucke werbewirksam herausgestellt - Forderungen, durch die sich verschiedene Schichten der ländlichen Gesellschaft, Baurschaft und Hindersessen, repräsentiert fühlen konnten bzw. sollten.

Andererseits steht außer Frage, daß die Hauptorganisatoren des bäuerlichen Widerstandes zumindest in der Anfangsphase im Milieu von Großbauern und gut situierten Handwerkern bzw. ‘Dienstleistern` wie Wirten und Müllern zu suchen sind, womit Parallelen zur Orientierung der sogenannten Voraufstände zu ziehen wären. Die Annahme jedoch, die Großbauern hätten sich der Kleinstellenbesitzer bei der Durchsetzung von Partikularinteressen lediglich bedient, hält den Ereignisabläufen nicht in jedem Falle stand. Neuere Forschungen haben nämlich versucht, die widersprüchlichen Phänomene weniger

\footnotetext{
${ }^{104}$ Rudolf HolBaCH, Formen des Verlags im Hanseraum vom 13. bis zum 16. Jahrhundert, in: Hansische Geschichtsblatter 103 (1985), S. 41-73, hier S. 60 f.

${ }_{105}$ Claus Peter ClASEN, Die Augsburger Weber, Augsburg 1981, S. 142.

${ }^{106}$ Ibid., S. 141, 149, 229 f.

${ }^{107}$ BLICKLE, Revolution (wie Anm. 1), S. 50; UlBRICH, Leibherrschaft (wie Anm. 12), S. 76.
} 
durch regionale als durch zeitliche Differenzierung besser aufeinander zu beziehen. Dabei haben sie an der Beobachtung angesetzt, daß sich die Ereignisse im Bauernkrieg an verschiedenen Orten in zwei Phasen einteilen lassen, von denen die erste eher von Demonstration und Verhandlung, die zweite dagegen von gewaltsamen Auftritten gekennzeichnet war ${ }^{108}$.

Gowind P. Sreenivasan hat im Anschluß an ältere Einschätzungen zum Bauernkrieg in Tirol die Phaseneinteilung für Ottobeuren dergestalt mit der sozialen Problematik verknüpft, daß er die erste, an ältere Konfliktmechanismen anknüpfende Verhandlungsphase von den herkömmlichen ländlichen Eliten dominiert sah. Für die zweite Phase, den Sturm auf das Kloster, konnte er nachweisen, daß weniger die bäuerlichen Eliten als kleine Landbesitzer und Angehörige der Unterschichten beteiligt waren ${ }^{109}$. Auch im Aufstand gegen das Kloster Ochsenhausen standen 1525, wie bereits Sabean herausgearbeitet hat, die Interessen der Tagelöhner an erster Stelle ${ }^{110}$. Grundlage für das eigenständige Auftreten der unterbäuerlichen Schichten ist zunächst eine große Zahl, ein weiteres Indiz dafür, daß die Bevölkerungsverluste des Spätmittelalters in den meisten Bauernkriegsgebieten längst kompensiert waren.

Für fränkische Weinbaugebiete sind die Abläufe genauer verfolgt worden. Großbauern organisierten die ersten Zusammenkünfte und Aktionen; Kleinbauern, Unterschichten und die große Zahl von Wanderarbeitern wurden nur unzureichend informiert und teils zur Teilnahme gezwungen. Je mehr im weiteren Verlauf der Verhandlungsweg verlassen wurde, um so mehr traten aber die Unterschichten in Erscheinung, um bei den zahlreichen gewaltsamen Aktionen, durch die der Bauernkrieg gerade in Franken gekennzeichnet ist, schließlich die Initiative zu übernehmen ${ }^{111}$, wobei auch wausgesprochene Habenichtse ${ }^{112}$ in die Führung aufrückten. Umgekehrt gab es Führungspersonen aus dem großbäuerlichen Milieu, die nach dem Krieg ähnlich wie einige Beteiligte aus dem Adel aussagten, sie seien zur exponierten Teilnahme gezwungen worden bzw. hätten anfänglich die Führung übernommen, um Schlimmeres zu verhindern ${ }^{113}$.

Nicht überall können die Bauernkriegsereignisse in zwei Phasen eingeteilt werden, und selbst wenn, dann ist ein schematischer Bezug der Verhandlungsphase auf Interesse und Organisation der Oberschichten und der gewaltsamen Phase auf die Unterschichten selbstredend problematisch. Es gab auch unter den Rädelsführern, die aus der Oberschicht kamen, Personen, die gewaltsame Übergriffe favorisierten oder im Verlauf der Erhebung einen Radikalisie-

${ }^{108}$ SREENIVASAN, The Social Origins (wie Anm. 15), S. 56.

${ }^{109}$ Ibid., S. 57.

${ }^{110}$ SABEAN, Landbesitz (wie Anm. 12), S. 42-44.

${ }^{111}$ VICE, Leadership (wie Anm. 64).

112 ENDRES, Probleme des Bauernkriegs (wie Anm. 3), S. 100.

${ }^{113}$ FrANZ, Bauernkrieg (wie Anm. 7), S. $217 \mathrm{f}$. 
rungsprozeß durchmachten. $\mathrm{Zu}$ ihnen gehören bekannte Gestalten wie Erasmus Gerber aus Molsheim im Elsaß und Jakob Rohrbach aus Böckingen bei Heilbronn. Sie - oder auch andere Teilnehmer - hatten mit einzelnen Herrschaftsträgern oft alte Rechnungen ${ }^{114}$ offen, die sie im Bauernkrieg zu begleichen hofften.

Die charakteristische Teilnahme von Angehörigen der Unterschichten an den genannten spektakulären Aktionen einer zweiten, gewaltsamen Aufstandsphase sollte also nicht vorschnell verallgemeinert werden. Für die erwiesenen Fälle stellt sich außerdem die Frage nach den Motiven der Unterschichten für ihr gewaltsames Aufbegehren, die bei Sreenivasan eher im Ungewissen bleiben. Möglicherweise lassen sie sich - wenn auch sicher nur partiell - durch eine Art Gegenprobe erhellen, eine nähere Betrachtung des besonderen Verhältnisses großbäuerlicher Vertreter $\mathrm{zu}$ den Trägern der Grundherrschaften. Zwar sollten auch in dieser Hinsicht Verallgemeinerungen vermieden werden, in zahlreichen Territorien des Südwestens stellt sich das Verhältnis aber so dar, daß die größeren Bauern durch ihren Landbesitz (wenngleich sich im Vorfeld des Bauernkrieges Konflikte über die rechtliche Ausgestaltung entwickelt hatten) und durch die Ausübung dörflicher Ämter (wenngleich sich erhebliche Spannungen an der Kompetenzausübung entzündeten) mit der Grundherrschaft verbunden waren.

Obwohl von Ort zu Ort Unterschiede bestanden, war die Bindung an die Herrschaft v.a. bei der Ämtervergabe auch im Südwesten oft enger, als die eingangs dargestellten, in den Rahmen einer réaction seigneuriale einzuordnenden Kompetenzstreitigkeiten zu erkennen geben. In der Reichsabtei Ottobeuren z.B. wurden die dörflichen Ämter vom Abt ohne Mitwirkung der Gemeinden besetzt und waren allein für Lehensträger des Klosters zugänglich, womit nicht nur Unterschichten, sondern auch die Lehensinhaber anderer Grundherrschaften ausgeschlossen waren ${ }^{115}$. Im Umkreis des Schwarzwaldes war der Ämterzugang noch im 18. Jahrhundert in gleicher Weise geregelt, und es gibt Indizien, daß es zumindest in einigen Territorien im Schwarzwald und im Bodenseebereich zur Bauernkriegszeit ähnlich war, v.a. dort, wo Klöster ähnlich wie Ottobeuren ihre Grundherrschaft zum Aufbau eines Territoriums genutzt hatten ${ }^{116}$. Im Grunde genommen hatte sich in diesen Territorien auf lokaler Ebene eine Struktur herausgebildet, die der Erblehensschulzenverfassung östlich der Elbe ähnlich war.

Wie nahe sich Herrschaft und bäuerliche Führungsschichten dort kommen konnten, ist z.B. daran abzulesen, daß im schlesischen Peterwitz 1555 der Gutsherr sein Bargeld dem von Bauern administrierten Kirchenkasten anver-

\footnotetext{
${ }^{114}$ Ibid., S. 189.

${ }^{115}$ SREENIVASAN, Ottobeuren (wie Anm. 12), S. 44.

${ }^{116}$ BADER, Studien (wie Anm. 35), S. 288f.
} 
traute ${ }^{117}$. In Schleswig-Holstein war es bis ins 18. Jahrhundert nicht ungewöhnlich, daß Gutsherren die Patenschaft für Kinder aus der bäuerlichen Oberschicht, v.a. der Schulzen, übernahmen ${ }^{118}$. Daß umgekehrt ein Schulze seinen zukünftigen Gutsherrn, einen kleinen Moltke, aus der Taufe hob, ist allerdings nur für das Mecklenburg des frühen 16. Jahrhunderts verbürgt ${ }^{19}$. Sicher war das Einvernehmen dort weniger ausgeprägt, wo die Gutsherrschaft in ihrem Expansionsdrang auch auf große Bauern wenig Rücksicht nahm. Insofern kann die in Einzelfällen dokumentierte kooperative Beziehung von bäuerlichen Amtsträgern und Herrschaft nicht als Grund dafür angefügt werden, daß es im Osten - mit Ausnahme des ostpreußischen Samlandes ${ }^{120}$ - zur Bauernkriegszeit nicht zu nennenswerten Konflikten in der Fläche kam.

Außerdem sind enge Verbindungen zwischen Großbauern und Grundherren auch für die Bauernkriegsgebiete nachzuweisen. Noch im späten 16. Jahrhundert traten einzelne Bauern als Bürgen oder sogar als (Mit-)Vormünder ihres (künftigen) Grundherm in Erscheinung ${ }^{121}$. Freilich stellt sich auch für diese Fälle die Frage, wie repräsentativ sie sind. Im oberschwäbischen Ummendorf bei Biberach trug eine Besonderheit der geistlichen Grundherrschaft dazu bei, $\mathrm{da} \beta$ das Verhältnis zu den Bauern eher durch flache Hierarchien gekennzeichnet war. Der größte Teil der Bauern hatte prächtig mit dem Prämonstratensermönch Jacob Murer harmoniert, der seit 1499 ihr Pfarrer war. Murer vermittelte in Streitigkeiten mit dem Kloster, die sich bereits an der Wende zur Neuzeit ergaben, verlieh im Dorf Geld und Getreide, saß mit den Bauern in den beiden Wirtshäusern und stand auch für Patenschaften zur Verfügung ${ }^{122}$. 1523 wurde Murer zum Abt des Klosters Weißenau und damit zum Landesherrn der Ummendorfer Bauern gewählt. Im Bauernkrieg jedoch hielten die Verbindungen Murers in die dörfliche Gesellschaft hinein den Interessengegensätzen und der Dynamik der Ereignisse nicht stand. Es gelang ihm nur kurzfristig, die Aufständischen, die ihm persönlich bekannt waren, durch begütigende Ansprachen und die Einladung in die Wirtshäuser, wo er Brot und Wein spendierte, zu besänftigen. Wenig später schlossen sich auch die Ummendorfer den Aufständischen an und nahmen an der Plünderung des Klosters teil.

${ }^{117}$ Waldtraut MEYER, Gemeinde, Erbherrschaft und Staat im Rechtsleben des schlesischen Dorfes vom 16. bis 19. Jahrhundert, Würzburg 1967, S. $74 \mathrm{f}$.

${ }^{118}$ Jan KLUSSMANN, Lebenswelten und Identitäten adliger Gutsuntertanen. Das Beispiel des östlichen Schleswig-Holstein im 18. Jahrhundert, Frankfurt a.M. 2002, S. 174-178.

${ }^{119}$ Ernst MŬNCH, Herrschaftsstreit in den Augen der Gutsuntertanen: Das Beispiel Toitenwinkel bei Rostock, in: Jan PETERS (Hg.), Gutsherrschaftsgesellschaften im europäischen Vergleich, Berlin 1997, S. 343-350, hier S. 347.

${ }^{120}$ Heide WUNDER, Der samländische Bauernaufstand von 1525, in: WOHLFEIL, Bauernkrieg (wie Anm. 3), S. 143-176.

${ }^{121}$ BADER, Studien (wie Anm. 35), S. 290.

${ }^{122}$ Gunther FRANZ, Werner FleISCHHAUER, Jacob Murers Weißenauer Chronik von 1525. Text und Kommentar, Sigmaringen 1977, S. 28. 


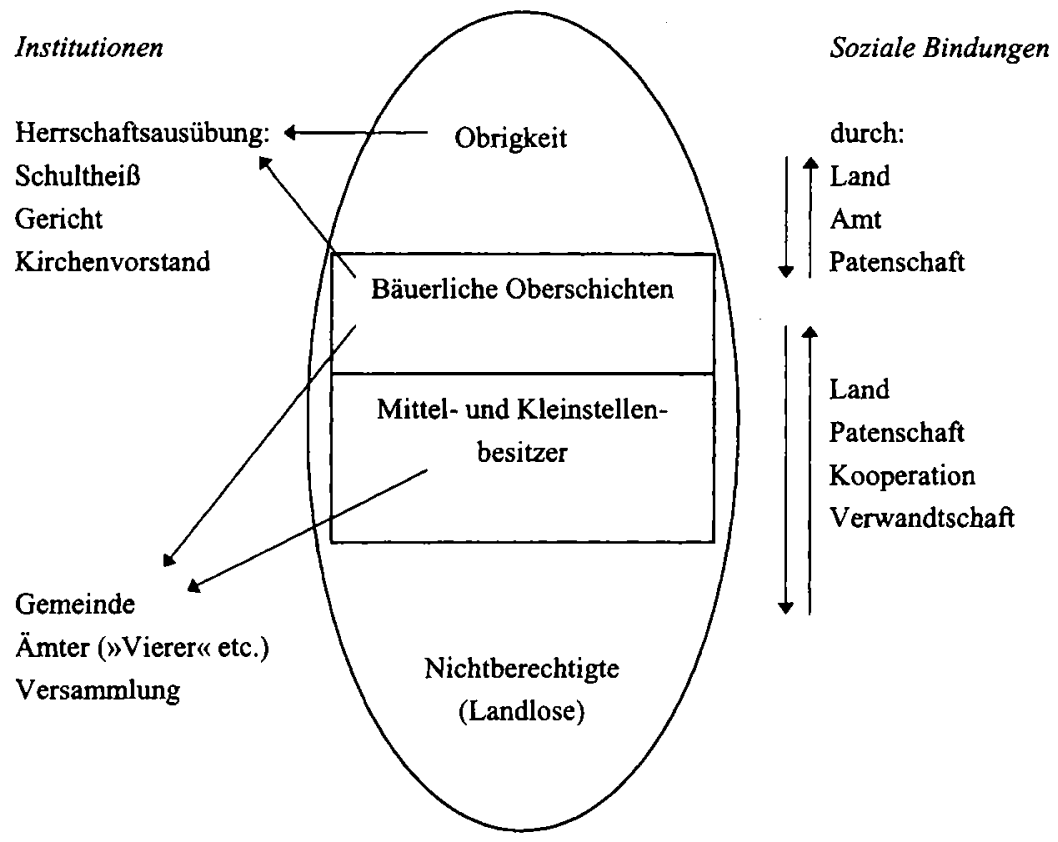

Institutionen und soziale Bindungen: Lokale Gesellschaft (rund)/Gemeinde (Viereck)

In normalen Zeiten reichten die Bindungen jedoch aus, die institutionelle Position zwischen Herrschaft und Untertanenschaft abzusichern, die von den dörflichen Amtsträgern - meist Vollbauern - eingenommen wurde, zumindest in Phasen, in denen auch wirtschaftliche Interessenkonvergenz bestand ${ }^{123}$. Dies war zu Beginn der Neuzeit zwar dadurch in Frage gestellt, daß die Obrigkeiten auf die Steigerung von Abgaben hinarbeiteten, grundsätzlich bestand aber die Einsicht, daß die großen Bauern nicht nur als politische Vertreter der Herrschaft in der Gemeinde gebraucht wurden, sondern daß ihre Höfe zugleich das wirtschaftliche Fundament der kleinen Landesherrschaften bildeten.

Betrachtet man die Machtverhältnisse auf lokaler Ebene, kann der Bauernkrieg zumindest in einer ersten Phase als Versuch bäuerlicher Eliten verstanden werden, materielle und administrative Positionen, die sie im Horizont der Auflösung von Villikationen und der Wüstungsperiode - die allerdings in Südwestdeutschland und Franken nicht überall katastrophalen Charakter annahm - errungen hatten, gegen eine réaction seigneuriale zu verteidigen, die sich vor dem Hintergrund von zunehmenden Bevölkerungszahlen und spürbaren Ressourcenverknappungen besonders in den von der Pest des 14. Jahr- 
hunderts weniger stark betroffenen Gebieten Süddeutschlands besser als ähnliche Versuche des 15. Jahrhunderts entfalten konnte. Zur Zuspitzung trug bei, daß sich auch die bäuerliche Position nicht allein als rreaktiv kennzeichnen läßt. Den Schub, der von der frühen Reformation ausging, wollte man dazu nutzen, bereits errungene Verfügungsrechte über die Ortskirche durch die Forderung nach freier Pfarrerwahl abzusichern und auszubauen. Teilweise kollidierten die Bestrebungen der Vollbauern mit den Interessen der unterbäuerlichen Schichten, z.B. auf dem Gebiet der Kontrolle über die Allmende. Anfangs war offenbar die Überzeugung verbreitet, daß die Differenzen zwischen Vollbauern und Obrigkeiten auf dem in den Jahren zuvor vielfach erprobten Weg von Verhandlung und Schlichtung beigelegt werden könnten.

Allerdings brauchten die Vollbauern die größer werdende Masse ihrer armen Brüder, Cousins, Schwäger und Schwiegersöhne, um ihren Forderungen gegen die Grundherrschaft Nachdruck zu verleihen. Dies konnte dazu führen, daß diese selbst die Chance ergriffen, ihren Unmut gegen Grundherrschaft und Leibeigenschaft zu artikulieren. Die Hemmschwelle für ein gewaltsames Vorgehen war für sie - das ist das Ergebnis der Gegenprobe - offenbar niedriger, da sie in viel geringerem Maße als die dörflichen Eliten emotionale und institutionelle Bindungen an die Grundherrschaft eingegangen waren. Es wird jedoch noch viel Arbeit zu leisten sein, bis die Motive genauer faßbar werden und allgemein das Verhältnis von sozialen Grundlagen und Ereignisfolge im Bauernkrieg in seinen vielfältigen Facetten besser begriffen und auf einer breiteren regionalen Grundlage dargestellt ist.

Voraussetzung für eine solche Darstellung ist nicht nur die Berücksichtigung der regionalen Unterschiede, sondern auch der internen Diversität, durch die sich die Agrar- und Sozialstrukturen in zahlreichen vom Bauernkrieg erfaßten Gebieten auszeichnen. Es ist kein Zufall, daß der Bauernkrieg nicht in den weitgehend durch Ackerbau und Tierhaltung geprägten Regionen Norddeutschlands stattfand, sondern in den städtereichen und diversifizierten Regionen der Oberrheinlande, Schwabens, Frankens und Thüringens, wo Wein- und Gemüsebau, Faser- und Färbepflanzen und nicht zuletzt die protoindu-strielle Textilherstellung zahlreichen Kleinstellenbesitzern und Landlosen eine Existenzmöglichkeit gaben. Ihre Lebensweise war in jedem Fall prekär und setzte ein hohes $\mathrm{Ma} \beta$ an Flexibilität und Mobilität voraus, die über den engen Horizont des Dorfes hinaus führte, sei es, daß sie als Pendelarbeiter in Städten bzw. benachbarten Bauerndörfern oder als Wanderarbeiter im Weinbau tätig waren, daß sie Flachs, Wein oder Gemüse auf kleinen Parzellen anbauten, sich ein Stück Vieh teilten, um es auf der Allmende weiden zu lassen, oder sich in Städte begaben, um Rohstoffe (z.B. Baumwolle) für ihre hausindustriellen Tätigkeiten abzuholen bzw. textile Halbfertigprodukte zu verkaufen. Beschränkungen - sei es durch Leibeigenschaft und Todfall, sei es durch die Sperrung der Allmende - trafen diese Menschen in ihrem 
Lebensnerv. Nicht zuletzt für eine Einordnung der Aufstandsbewegung in gesamtgesellschaftliche Perspektiven (»Revolution des gemeinen Mannes in Stadt und Land«) verspricht eine Analyse der Aktivitäten dieser mobilen Bevölkerungsgruppen weitere Einsichten.

In der zweiten Hälfte des Jahrhunderts, als die Zahl der Landlosen und Landarmen geradezu exponentiell zunahm, bahnte sich eine Verschiebung von Orientierungen und Bindungen an. Gemeinden und Verwandtschaften, die zuvor - wenn auch nicht überall - die armen Leute integriert hatten, sahen sich in starkem Maße überfordert. Vielerorts begannen Hexenjagden die soziale Kohäsion der Dörfer, das nachbarliche Zusammenleben, zu ruinieren. Die Obrigkeiten stützten sich zwar noch immer im wesentlichen auf die besitzbäuerlichen Schichten im Dorf, man begann jedoch auch paternalistische Strategien zu entwickeln, z.B. Steuererleichterungen, Kreditvergaben und Marktregulierungen, die die Existenzweise der Unterschichten erleichtern sollten. Es fehlen allerdings Studien, die diese erst exemplarisch dargestelten $\mathrm{Zu}$ sammenhänge ${ }^{124}$ vertiefen und vielleicht auch der Frage nachgehen, ob Erfahrungen aus dem Bauernkrieg zu dieser Umorientierung auf Seiten der Obrigkeiten beitrugen.

${ }^{124}$ RoBISHEAUX, Rural Society (wie Anm. 102), S. 167-173. 
